\title{
Intralesional Injection of Adalimumab for Anal Stricture in Pediatric Crohn's Disease: A Therapeutic Option?
}

ISSN: 2637-7632

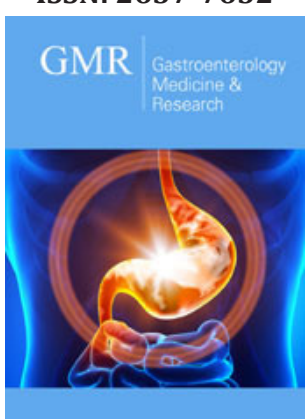

*Corresponding author: CamachoGomez S, Baylor College of Medicine at The Children's Hospital of San Antonio, San Antonio, USA

Submission: 慍 May 13, 2020

Published: 海 May 21, 2020

Volume 4 - Issue 4

How to cite this article: Camacho-Gomez S, Javier Monagas, James Meredith Noel. Intralesional Injection of Adalimumab for Anal Stricture in Pediatric Crohn's Disease: A Therapeutic Option?. Gastro Med Res. 4(4). GMR.000595. 2020.

DOI: 10.31031/GMR.2020.04.000595

Copyright@ Camacho-Gomez S, This article is distributed under the terms of the Creative Commons Attribution 4.0 International License, which permits unrestricted use and redistribution provided that the original author and source are credited.

\section{Camacho-Gomez S*, Javier Monagas and James Meredith Noel}

Department of Pediatrics, Baylor College of Medicine at The Children's Hospital of San Antonio, USA

\begin{abstract}
The symptomatic stricturing Crohn's disease is incapacitating and usually associated with therapeutic failures requiring surgery. We herein described a 13-year-old Caucasian male evaluated for a second opinion in the pediatric gastroenterology clinic for an anal Crohn's stricture. The patient underwent serial anal dilations followed by intralesional injection of the adalimumab. In spite of a wide range of therapeutic options for the management of Crohn's disease, the effectiveness of serial adalimumab intralesional injection in anal strictures to maintain anal patency has not been reported. We described the use of intralesional adalimumab to maintain the patency of the anal canal.
\end{abstract}

Keywords: Adalimumab; Anal stricture; Crohn's disease; Perianal disease; Anti-Tumor necrosis factor; Anal canal

Abbreviations: Crohn's Disease (CD); Anti-Tumor Necrosis Factor Alpha (anti-TNF); Subcutaneously (SQ)

\section{Introduction}

13-year-old Caucasian male with CD seen in the Gastroenterology clinic for a second opinion. He was previously diagnosed with severe anal stricture and active inflammation at the terminal ilium and all segments of the colon at 10 year of age. He experienced painful and difficult defecation despite dietary modification, laxatives, loose consistency stools, and SQ adalimumab therapy. He underwent endoscopic dilations and Hegar dilations for his strictured anal canal. He was also treated topically with triamcinolone and Mitomycin $\mathrm{C}$ without significant improvement and a permanent diverting colonic ostomy was advised. His previous treatments had included Infliximab, which failed to obtain remission and he developed antibodies to this anti-TNF. He then was treated with adalimumab (40mg SQ weekly) but despite excellent compliance for a year, he did not achieve therapeutic blood levels and no alleviation in his anal disease. He did not develop antibodies to adalimumab.

On presentation to our clinic, he was underweight with a BMI of $16.5\left(17^{\text {th }}\right.$ percentile). The anal exam was significant for an erythematous perianal rash (Figure 1A) and a rectal exam noted a firm stricture $1 \mathrm{~cm}$ from the anal verge, which was approximately $4 \mathrm{~mm}$ in diameter (Figure 2A). No other small intestine inflammation, stenosis or fistula were appreciated on a magnetic resonance. He underwent stepwise Hegar dilation, with an initial 4 French dilator. Once the canal was dilated to 18 French (Figure 2B), a colonoscopy was performed. His adalimumab level was $5 \mu \mathrm{g} / \mathrm{mL}$ which is sub-therapeutic. We added oral methotrexate $15 \mathrm{mg}$ weekly $\left(15 \mathrm{mg}_{\text {per }} \mathrm{m}^{2}\right.$ ) given orally. He was noted to be in clinical and histological remission from his colonic and terminal ileal disease, but a refractory anal stricture persisted (Figure 2C). 

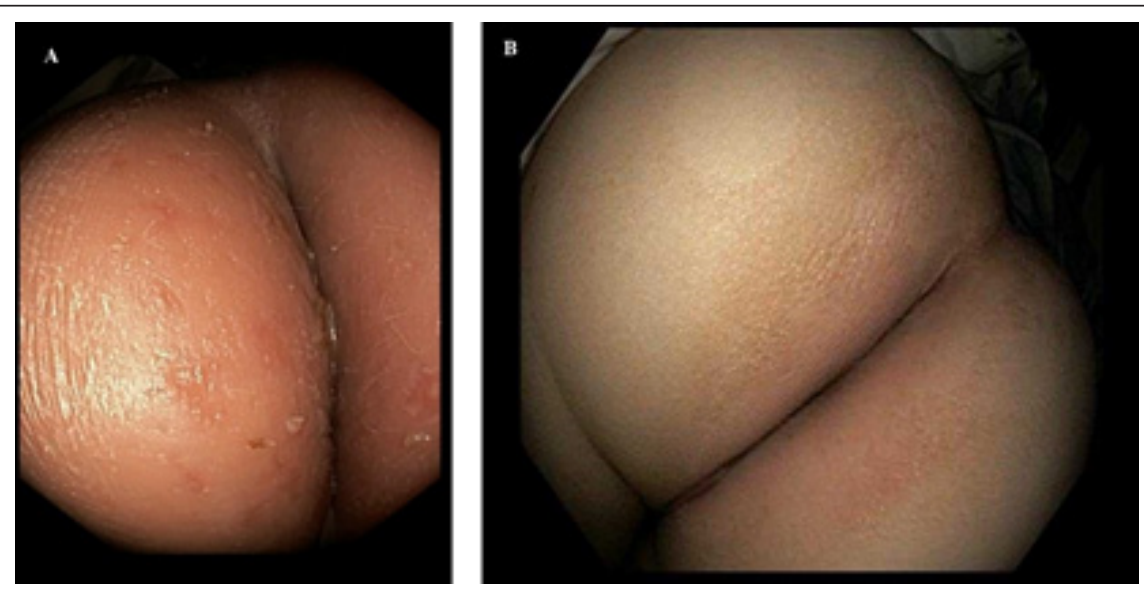

Figure 1: Perianal Crohn's Rash: A. Erythematous perianal rash. B. Perianal rash is resolved.

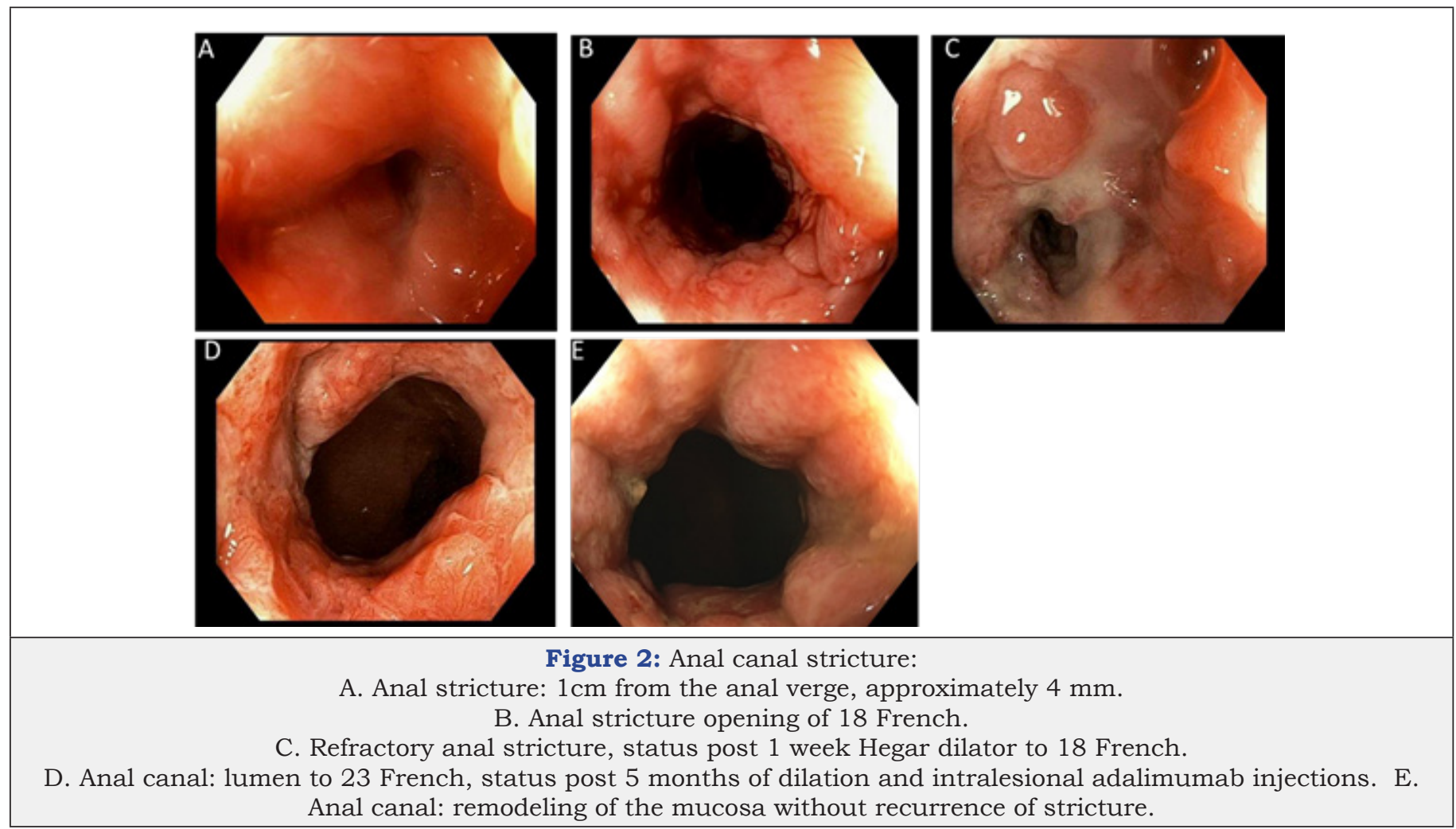

With a patent anal canal we began weekly dilatations and since this therapy had previously failed to maintain anal patency we added intralesional injections of adalimumab. Parents and patient agreed to try intralesional adalimumab injections, based on previous case reports of effective use of intralesional Infliximab with stricture dilation $[3,4]$ and signed informed consent. We used 40 milligrams of adalimumab with 10 milligrams injected into the anal stricture at $2,4,8$, and 10 o'clock for a total dose of 40 milligrams injected into the stricturing Crohn's disease. Classic Hegar dilation was used with each treatment, progressing up to 23-French. He received weekly treatment for a month and then every 2 weeks for a month with intralesional injection combined with dilation. As patency was ow being maintained we administered SQ injections to once a week except for the fourth week when we delivered a perianal intralesional injection into the fibrotic stricture. Attempts to lengthen the intralesional injections to every 6 weeks resulted in narrowing of the anal canal to 18 French and difficulty defecating.

With the addition of Methotrexate and the weekly adalimumab injections we achieved a therapeutic adalimumab level of $15.1 \mu \mathrm{g} /$ $\mathrm{mL}$. The anal stricture reached a stable diameter of 23 French. We were now able to administer the monthly intralesional adalimumab injection without further anal dilations. The anal canal remained patent 23 French. In addition to monthly intralesional injections, subcutaneous adalimumab were continued weekly, except for the week he received the intralesional injections. The insurance approved for flexible sigmoidoscopy and dilation as part of his anal stricture therapy every six to eight weeks.

On initial laboratory evaluations C-reactive protein, erythrocyte sedimentation rate were elevated. These laboratory 
values normalized with the attainment of therapeutic adalimumab levels (Table 1). Over the 5 months of therapy, he gained $7 \mathrm{~kg}$ with a normal BMI (50 percentile) with no further difficulties defecating. The perianal rash is resolved (Figure 1B) and the anal canal is maintained the lumen at 23 French without recurrence of structuring (Figure 2D) and is developed remodeling of the mucosa lining and epithelium at 2 years into therapy and the intralesional injection of adalimumab has decreased to every 8 weeks (Figure 2E).

Table 1: C-reactive protein, erythrocyte sedimentation rate (ESR) and calprotectin improvement over the course of therapy.

\begin{tabular}{|c|c|c|c|}
\hline & Initial & 5 Months & 2 Years \\
\hline C-reactive protein $(\mathrm{mg} / \mathrm{L})$ & 7.8 & 0.28 & 0.06 \\
\hline ESR $(\mathrm{mm} / \mathrm{hr})$ & 21 & 9 & 8 \\
\hline Calprotectin $(\mu \mathrm{g} / \mathrm{g})$ & 522 & 523 & 53 \\
\hline
\end{tabular}

\section{Discussion}

Anal strictures in $\mathrm{CD}$ that are symptomatic are often incapacitating with the patient developing urgency, incontinence, tenesmus, infections and straining to defecate [2]. Given the transmural nature of $\mathrm{CD}$, these strictures affects all layers of the bowel wall. The pathology results from mesenchymal cell expansion and extracellular matrix accumulation [5]. Genetic markers have been proposed to predict stricture formation and early surgical recurrence in CD patients [5]. The management of CD patients has been rapidly evolving and for many years [1]. The fibrosing stricture was associated with therapeutic failure requiring surgery or endoscopic dilation [1]. Our patient underwent several endoscopic dilations with an assortment of dilations, combined with topical and systemic therapies. His next proposed course of management was proctocolectomy. Clinical remission, mucosal healing and appropriate growth are the main goal for pediatric population treatment [6]. Our patient has achieved the following goals of treatment with mucosal remodeling, attaining a normal BMI, and resolution of rectal mucosal disease on biopsy after instituting intralesional injection of adalimumab, and initiating methotrexate as an adjuvant therapy which improved his serum adalimumab levels.

Anti-TNF therapy along with immunomodulators are the best current therapeutic options for obtaining clinical remission and for preventing long-term therapeutic failure [6]. The combination of intralesional infliximab with endoscopic dilation has been shown to be effective in a small group of patients $[3,4]$, in maintaining anal canal patency in a patient refractory to medical therapy including systemic infliximab [3]. Adegbola et al. [7] in 2017 reviewed the effect of the local injection of anti-TNF for perianal fistulising Crohn's disease and did not find any severe adverse events [7]. The CREOLE study showed that intalesional adalimumab injection is effective for CD patients with symptomatic small bowel strictures [8]. To date no specific intestinal antifibrotic therapy exists, and perianal localization of Crohn's disease involves significant morbidity and affects quality of life [2]. Though studies are being conducted with intralesional adalimumab for intestinal and colonic strictures in $\mathrm{CD}$ as well as for perianal fistula, to our knowledge this is the first case of successfully treatment of an anal stricture with intralesional adalimumab in combination with anal dilation. The results may have occurred from reaching a therapeutic adalimumab level and the addition of methotrexate, but the potential for anti-TNFs to effect fibrosis was felt to contribute to the stable patency of his anal canal.

\section{References}

1. Keljo DJ, Markowitz J, Langton C, Lerer T, Bousvaros, et al. (2009) Course and treatment of perianal disease in children newly diagnosed with Crohn's disease. Inflamm Bowel Dis 15(3): 383-387.

2. McClane SJ, Rombeau JL (2001) Anorectal crohn' disease. Surgical Clinics of North America 81(1): 169-183.

3. Swaminath A, Lichtiger S (2008) Dilation of colonic strictures by intralesional injection of infliximab in patient with Crohn's colitis. Inflamm Bowel Dis 14(2): 213-216.

4. Teich N, Wallstabe I, Schiefke I (2017) Topic infliximab injection for refractory rectal stenosis in Crohn's disease: Long-term follow-up in two patients. Int J Colorectal Dis 32(9): 1289-1294.

5. Rieder F, Zimmermann EM, Remzi FH, Sandborn WJ (2013) Crohn's disease complicated by strictures: A systematic review. Gut 62(7): 10721084.

6. Aloi M, Nuti F, Stronati L, Cucchiara S (2014) Advances in the medical management of paediatric IBD. Nat Rev Gastroenterol Hepatol 11(2): 99-108.

7. Adegbola SO, Sahnan K, Tozer PJ, Phillips RK, Faiz OD, et al. (2017) Review of local injection of anti-TNF for perianal fistulising Crohn's disease. Int J Colorectal Dis 32(11): 1539-1544.

8. Bouhnik Y, Carbonnel F, Laharie D, Stefanescu C, Hebuterne X, et al. (2018) Efficacy of adalimumab in patients with Crohn's disease and symptomatic small bowel stricture: A multicenter, prospective, observational cohort (CREOLE) study. Gut 67(1): 53-60. 